\title{
Workshop Summary: AAPS Workshop on Special Dosage Forms-What's New with In Vitro Drug Release?
}

Vivian Gray

Dissolution Technologies, Hockessin, DE 19707
T he AAPS Workshop on Special Dosage FormsWhat's New with In Vitro Drug Release? took place at the AAPS Annual Meeting in Los Angeles, CA on November $7-8,2009$. This is my personal summary of each presentation and discussion, written from the perspective of a workshop attendee on a press pass from AAPS.

Day 1

Speaker:Vinod P. Shah, Ph.D.

\section{International Perspectives on the Drug Release of Special Dosage Forms}

This workshop was co-sponsored by FDA and FIP. This kick-off presentation introduced the workshop by providing background and objectives. The workshop focused on modified-release drug forms and included regulatory perspectives and specific

presentations for orally disintegrating tablets, chewable oral drug products, ophthalmic products, aerosol drug products, drug-eluting stents, and parenteral products including nanoparticles, microspheres, and liposomes.

Each product is classified (sections in each of Tiers 1-3), and tests including product quality tests (identity, strength, potency, and quality) and product performance tests (in vitro release) are carried out. Examples were given for product quality tests and product performance tests for each Tier 1 classification. The most complex test for each Tier 1 classification tends to be product performance in determining an in vitro release method that is predictive of in vivo performance is complex. Other complex initiatives exist for transdermal products such as leak testing, which is critical to ensure understanding of potential toxicology issues. USP Apparatus 5, 6, and 7 are generally sufficient for evaluation of the release of patches. The discussion of aerosol drug products highlighted the importance of particle size testing, and particles $<5 \mu \mathrm{m}$ are ideal for effective performance for this Tier 1 classification. Mucosal products have multiple applications, and much work has been performed around in vitro release testing for this Tier 1 classification. To date, none have been standardized and various apparatus have been utilized for analyses of this class of products (mini-paddles have been employed but are not standardized). There are many USP chapters readily available to provide insight to the testing of oral, aerosol, injectable, mucosal, and skin drug products. Some are finalized and many are in development phases.

FIP has committed to revise the publication "FIP/AAPS Guidelines for Dissolution/In Vitro Release Testing of
Novel/Special Dosage Forms" during the 2010 calendar year.

Speaker: Henk de Jong, Ph.D.

International Perspectives on the Drug Release of Special Dosage Forms-The European Pharmacopoeia

The EDQM and European Pharmacopoeia were defined. The EDQM is committed to applying ICH guidances. Thirty-seven countries are members of the European Union, and each is required to follow the European Pharmacopoeia. Any exemption from a Ph. Eur. requirement must be justified and approved. One key difference between the Ph. Eur. and the USP is that there are not specific monographs for dosage forms. Each dosage form is required to meet approved specifications by following general dosage form monographs. The $P h$. Eur. dissolution general chapter (2.9.3) is harmonized with the USP and JP on the majority of points for oral solid dosage forms. Apparatus 1-4 are defined in the Ph. Eur. The nonharmonized issues between compendia are as follows: Apparatus 3 is not accepted by $J P$, sequential testing is not accepted by $J P$, pooled samples are not accepted by $P h$. Eur., and calibrator tablets are not accepted by Ph. Eur.Ph. Eur. has an informational chapter regarding guidance on dissolution testing and list of media lacking. Ph. Eur. has a dissolution test for transdermal patches (2.9.4). A noted difference for this chapter is the defined skin (model) temperature that is lower than that in chapter 2.9.3.Ph. Eur. also has a chapter in progress for medicated chewing gums (2.9.25) that defines the mechanical kneading principle (not yet completely defined). There is one apparatus (mastication instrument) in the monograph and a second is under discussion. Ph. Eur. also has a chapter available for lipophilic solid dosage forms (2.9.42). Proficiency testing was summarized. This effort was carried out by the EDQM to look at consistency of dissolution testing versus pre-established criteria for six different studies. All have been completed over the past nine years, but none has been published externally. The study concluded that high percentages (77-96) of satisfactory results were obtained in the study. With the exception of the mesh-size issue for baskets (being evaluated for impact now), conventional dosage forms are well-defined with respect to dissolution testing. Proficiency testing further supports sufficiency of mechanical calibration. The main outstanding chapter for Ph. Eur. at this time is 2.9.25. An additional point that was discussed in the questions segment was a request to define how the Ph. Eur. will react 
to new chapters published by the USP.The goal is to maintain as much harmonization as possible and not to head back into the current harmonization project that is on-going for so many chapters.

\section{Summary of Questions}

The use of organics or enzymes is not defined or harmonized. Will this be identified as a condition when dissolution methods are harmonized? There will be a symposium as part of AAPS on the performance of alcohol studies with dissolution.

Additional explanation on failures for the proficiency test was requested. The explanation provided was that failures were investigated (equipment, protocol interpretation, etc.) and concluded before retesting.

A question of how stents and implants would be covered in the Ph. Eur. was posed. It was indicated that these will fall under devices, but would also be considered combination products. It will be useful to have standardization for these device requirements.

Speaker: J. Michael Morris

International Regulatory Views on Special Dose Forms

The necessity for sound science in dosage form design, in the manufacturing process, and in the simulation of in vivo performance by in vitro testing was emphasized. The state of harmonization between regulatory agencies and their requirements to follow ICH were summarized. ICH guideline topics as well as learning points from their development were provided. The list of priority harmonization chapters was included and their status was presented. The focus was then shifted to in vitro release and Q6A, where items not covered were identified. The importance of thorough development of methods with respect to Q8 (QBD) was emphasized. Submissions should include all relevant data for IVIVC or IVIVR. Determination of relationships/correlations is more complex with modified-release products. Different routes of administration and nanotechnology and liposomes were presented. The importance of determining the right method with appropriate specifications, no matter what formulation, was reiterated.

Speaker: Cynthia Brown

\section{In Vitro Release Testing of Special Dosage Forms Summary of $\mathbf{2 0 0 8}$ London Workshop}

This presentation provided high-level information for each modified-release formulation as presented in London in 2008. The FIP SIG was introduced, and a commitment was made to revise the 2003 publication of FIP/AAPS guidelines for in vitro release. Much progress has been made since the original publication in the area of modified-release dosage forms, and recommendations/ standardization would be beneficial. The goal is to provide standards, where acceptable, for in vitro release testing of modified-release products. Modifications of standard Apparatus 2 and different conditions for testing orally disintegrated tablets and nasal dosage forms, lozenges, patches, and chewable gums were presented. Stents and microspheres were summarized based on the London conference. Recommendations from the conference were presented for modified scaling, modified temperature, and modified apparatus. Apparatus 4 and its application with microspheres were identified. It is necessary to optimize the in vitro method to reflect in vivo activity appropriately and discriminate between manufacturing changes. Recommendations for inhalation products and suppositories were presented and include impactor testing and modified dialysis tubing method, respectively. Dissolution is necessary to assess batch-to-batch quality, and methodologies need to be simple, reliable, and reproducible.

Speaker: Lucinda Buhse, Ph.D.

\section{Orally Disintegrating Tablets or Orodispersible Tablets}

Definitions from both the FDA and Ph. Eur. for orally dispersible tablets were presented, and identification of dosage forms not within the classification was provided. There are advantages to orally disintegrating tablets, but many issues are observed due to the specialized formulation. These tablets should be assessed similarly to other tablets, but disintegration testing may have greater value. Disintegration and dissolution testing should be used to guide development and to understand potential impact of manufacturing changes and IVIVR/IVIVC. Acceptance criteria should be based on scientific merit and statistical evaluation. In later stages, these tests should be used as a quality control tool. Development of a dissolution method may be complex due to formulation-driven limitations such as rapid disintegration or necessity for sinker use. In vivo versus in vitro comparisons for disintegration are complicated because the in vivo characteristics can only be measured with respect to patient input. Standards for in vitro testing were not intended for ODT and will likely not perform appropriately without modification. Examples of issues with measurement of disintegration (both in vivo and in vitro) were presented, and the potential importance of texture was identified. Modified Apparatus 2 and CCD cameras were presented as options for measurement of disintegration. Both dissolution and disintegration are important for ODTs, and additional development will be necessary to ensure proper comparisons for disintegration.

Speaker: Gordon McKay, Ph.D.

\section{In Vitro Evaluation of Chewable Oral Drug Products}

This presentation summarized in vitro testing for gum-based and chewable tablets. The introduction summarized various in vitro techniques utilized to analyze specialized dosage forms. The focus then turned to mastication instrumentation for analysis of chewable products. Chewable products offer advantages based on patient base and mode of delivery. The success of 
Nicorette including the complications of ex vivo chew-out studies (design, patient selection, training of candidates, lack of availability of objective/QC tool, etc.) was highlighted. The mastication instrument (described in Ph. Eur. as not commercially available) as well as the Erweka system (DRT-6) were presented, including videos of the actual operation (calibration and operation of DRT-6). One of the biggest challenges with the instruments is the standardization of the jaws and getting them to be similar to chewing. Chewing frequency of 40 chews per minute has been determined to be adequate (a question was asked if this was published as it would be an important reference for further work). The rotational angle of the instrument can be changed anywhere between 0 and $180^{\circ}$ and the angle of $20^{\circ}$ is noted as acceptable. The combination of jaw distance and rotational angle must be optimized, because these properties impact product release. Temperature robustness has also been evaluated and was robust between 35 and $37^{\circ} \mathrm{C}$, but not up to $39^{\circ} \mathrm{C}$. Trouble-shooting of the instrument settings was demonstrated. For example, when the jaw distance is too high, additional scatter may be observed. A graduate (engineering) project is to evaluate the impact of rheology of gum products on release. For example, different flavoring requires different chewing strength for the same amount of product release. One other instrument component that impacts robustness of in vitro compatibility is netting. Netting is required to prevent fragmentation of gum products, and a change in type impacts the effective distance between upper and lower jaws and thus the robustness of the mastication test. Viscosity and density characteristics have been evaluated as part of setting determinations. With the correct settings and the appropriate method development, inter-run variance can be minimized. The mastication instrument was also evaluated for chewable tablets because it may provide a faster testing alternative to the currently prescribed 14-h Apparatus 1 or 2 methods.

\section{Summary of Questions and Comments:}

- A question about aerosol requirements/Davis dissolution: Is this the right test? Are we measuring enough? A dissolution test has been developed in Austin, Texas, that may have applications for modified-release products

- Has there been any concentration on oral films? This should be considered; ODT guidance may apply.

- The suitability of oral tablets/pediatric dose was asked. This has been well-established. The FDA would currently require clinical trials as with any new indication or line extension. There should be a different evaluation to assess swallowed versus chewed, and bioequivalence would be necessary. However, there is no difference between pediatric and adult chewables.

- A comment was made that the multi-stage impactor is not necessary for inhaled products. Better results would be obtained with two-stage only because the goal is not to impact all stages, only more of the final stage for in vitro.

- A question was asked if anyone has been successful in replacing in vitro release testing with particle size or disintegration testing (i.e., justify with QbD?) The general response was that testing should be justified with the appropriate rationale.

- A comment was made that beaker and basket size must be specified in the dossier for disintegration.

- Applications for Apparatus 3 for chewable tablets were discussed (different volumes, addition of glass beads, fiber-optic probes, etc.) The general issue is robustness. Mini-paddles have also been evaluated but are not yet specified in any guidance.

Speaker: Larry Stevens, M.S.

\section{Dissolution: Ophthalmic Inserts a High-Performance Approach}

Approaches for in vitro testing of ophthalmic inserts including multiple instruments and techniques with relevance to biologics, physiochemical, and regulatory were presented. Two types of testing cells were presented including the gel cell and the suspension cell. The applications vary depending on the type of insert and the dose site. One complication for in vitro test design is turbulence and flow velocity (and how it relates to in vivo conditions). A diffusion-controlled (closed) system was presented along with the effects of stirring and hydrodynamic vectors. This was then compared to a convection diffusion-controlled open system. Then the application of USP type 4 cell with the presence of glass beads as compared with the modified flow-through cell (adjusted geometry to improve sensitivity, thus measuring low concentrations) was presented.The micro dissolution method should be optimized for stir rate and flow. A high performance flow-through system was developed to improve productivity and reproducibility of analyzing low-dose devices. Other technologies such as NIR imaging were discussed to demonstrate their ability to evaluate drug homogeneity. To best evaluate in vitro release for special dosage forms, modification of traditional dissolution approaches will be necessary.

Speaker: Kent Alleman, Ph.D.

\section{Case Studies-Drug Release Measurements on Ophthalmic Dosage Forms}

Complications to ocular drug delivery were summarized, and drug delivery options were presented. Standard dissolution tests are not effective for evaluation of ophthalmic dosage forms because they are designed to simulate the GI tract. USP Apparatus 4 has been employed with challenges such as scale and clogging filters on the exit cells. Specialized tests are necessary. Depending on the dosage forms, different tests may be required (i.e., solutions, ointments, suspensions) Multiple case studies were presented to demonstrate some possibilities for in vitro tests for ophthalmic products. The first case study for ophthalmic suspension was presented. For suspensions, 
the main factor is particle size. While dissolution may discriminate between particle size differences, the more appropriate measurement is particle size. The second case study was ion-exchange resin suspension. The modified instrumentation was utilized to determine the impact of tear fluid on product release. While this is not suitable for a true release test, it was able to provide valuable information that tear production is a rate-limiting step for drug release. The $\mathrm{QC}$ test for particle size distribution may not provide as much information, but will discriminate product changes. The third case study presented erodible gels and gel-forming solutions. In this formulation, drug is released slowly as the gel is eroded by tears. A flow-through cell was utilized to simulate tear fluid and measure the drug in the effluent. While this test may not provide IVIVC information, it is useful to measure erosion rate and to compare formulations. The final case study described measurements of implants. Wet testing was performed (at a micro scale) to analyze release and to correlate to in vivo explants. The QC release test was set as a dry storage release rate measurement. IVIVC is complicated because it is also necessary to model clearance from the eye. However, in vitro data for ocular products are complicated to obtain.

Speaker: Jolyon Mitchell

\section{Performance Tests for Aerosol Products: Nasal}

Regulatory requirements have been established for inhalation and nasal products. Section 4.2.2 was noted as a reference for tests to characterize nasal products (although not all tests are necessary for all types of nasal products). Six distinct categories were shown, and it is necessary to know the form to interpret the guidance. Depending on regulatory governing body, established requirements may differ. Droplet size distribution is critical for appropriate delivery. Draft BA/BE Guidance was presented; Ph. Eur. and USP were compared (based on current state of revision). The current test strategy may be telling us more than needed. Nasal testing really requires a two-stage impactor ( $>10$ versus $<10 \mu \mathrm{m}$ ). There are challenges to the design of the current impactor, namely the angle of the "throat." In addition, the flow rate may impact measurements. Abbreviated impactors are becoming available to make measurements more simple and rapid. In addition to mass fraction analysis, size characterization is important for nasal products. Currently, laser diffractometry is the only method available (and is specified in the FDA BA/BE draft guidance). This is not a particle-counting method. Monodisperse versus polydisperse particles were differentiated (homogeneous versus heterogeneous sprays). LD was compared to PSD. A newer technique is time-dependent LD, which can tell how well the delivery system is working throughout its life cycle. Spray imaging is also being employed and provides simultaneous measurements of spray-pattern properties (time evolution, particle distribution, and divergence angle). Another technology employed for nasal drug delivery systems is Raman chemical imaging, which provides the benefits of Raman shifts specific to APIs and excipients (may not be detected by LD). RCI may be beneficial in determining $\mathrm{BE}$ between generic and innovator drugs. The final measurement that was presented was spray-impaction force, which may be perceived by the patient but may not have biological relevance. Further work is necessary to standardize test requirements for aerosol products (especially to differentiate between oral and nasal products).

Speaker: Anthony Hickey, Ph.D.

\section{Performance Testing of Dosage Forms-Inhalation Aerosol}

This presentation provided additional information on inhalation aerosols. Background was provided to introduce the hierarchy of inhalation products (lung and nose). In all cases, performance determinations are made based on particle size and delivered dose as well as dissolution. Considerations are necessary for delivered-dose uniformity as well as aerodynamic particle size analysis. The impactor that is used to analyze particle size has been improved and is now commercially available. While a logarithmic calculation is sometimes performed to analyze data, regulatory bodies are more interested in deposition on stages because of the nonlinear plots and poor logarithmic correlation. Dilemmas exist for analysis of inhalation products because of the inability to calibrate the impactor consistently and to standardize product analysis (analysis depends on the "device" used to disperse the product). Also, profile comparisons are complex because there are currently no statistical approaches to provide a discriminating tool between one stage and another. An earlier presenter (Jolyon) recommended grouping stages instead of separating each. Grouping would be better for statistical analysis and generation of more "real" results. The final dilemma is IVIVC, because it is not determined to be applicable to the impactor analysis. For example, the decrease in linear velocity from top to bottom of the impactor is a typical QC tool but is not modeled for IVIVC. Particle size testing for DPIs may not accurately simulate use by a patient. There are also other factors for formulation development that may be easy to model in vitro, but may not be practical in vivo. Examples were presented where in vitro testing may be over discriminating. As a result, in vitro release testing may not be the most value-added test for aerosol products (immediate release). If controlled-release products are introduced, an appropriate in vitro release test should be considered.

Speaker: Lori Alquier, Ph.D.

\section{Performance Tests for Drug-eluting Stents: Industry Perspective \\ For stents, chemistry dissolution is performed in $\mathrm{HCl}$.} The biological state differs-blood flow through the stent, the tissue surrounding the stent, and the healing process of the tissue in which the stent is implanted cannot be modeled in vitro. In vitro testing of stents had to move 
away from USP 1 to USP 4 and USP 7. USP 7 utilizes test tubes with elution media (dipping back and forth) and a stent holder that looks like paper clips. USP 4 is more of a model of the circulatory system $(25 \mathrm{~mL} / \mathrm{min}$ closed system) that can sample at different times. The stent is suspended into the cell. However, this is a static system, and the stent exists in an area of high turbulence. Therefore, there are many challenges to mimic the biological model through chemistry. A guidance for industry for drug-eluting stents is now available. It indicates that an in vitro release specification should be registered. Product should be tested over sufficient time with sufficient time points. The test should be a QC tool that is discriminatory and measures percent released versus unit time. The method should have the potential for validation and should correlate with the in vivo profile. It is critical to obtain many time points during development to appropriately select specification time points. Definition of beginning, middle, and end may be complex and should be scientifically justified based on the expected profile. The discriminating ability of the method should be demonstrated based on failure modes and effect analysis. Reporting assay and label claim may be performed as part of development. The in vitro test should correlate with the animal profile, and it is necessary to ensure that over-discrimination is not required. The method should be stability indicating for the drug as well as for polymer; however, if a different method is scientifically justified, this may be acceptable. The in vitro test method should be developed as early as possible to ensure appropriate discrimination while comparing to clinical data.

Speaker: Angelica Dorantes, Ph.D.

\section{In Vitro Release Testing of Drug-Eluting Stent Systems: A Regulatory Perspective}

Regulatory jurisdiction for drug-eluting stents falls with both CDRH and CDER. There are multiple drug-eluting stents approved and marketed today. Summaries of some of the approved products were provided. Depending on product development, the stent may not release all of the product (e.g., TAXUS is developed to release only 10-20\% of the embedded product). In each case, the matrix is developed and must be compared through in vitro testing and in vivo (may be explant) results. The coronary drug-eluting stent guidance for industry is still in draft form (nearly complete) and includes requirements for clinical pharmacology information and drug release kinetic information (both in vivo and in vitro). While animal studies are helpful during development and for establishment of in vitro test methods, IVIVR from animals (unless it is a validated animal model) is not acceptable for regulatory submission. IVIVR may be based on human data (noninvasive methods or explant data). It is recommended to develop the in vitro release test as soon as possible and to communicate with the agency throughout studies to ensure the correct information is collected. Come to a resolution on the in vitro release method, data needed, and acceptance criteria before submitting an application.
Acceptance criteria should be set based on the development and validation information for the in vitro test. The importance of a reliable, reproducible in vitro test was emphasized. The test should assure consistent product quality, measure product stability throughout shelf life, and predict the effect of process and formulation changes. If the test is well-developed, it may allow for use of the in vitro test in place of additional in vivo testing for formulation changes or other pre- or post-approval CMC-related changes. The final QC test should cover at least $80 \%$ released or an appropriate plateau. Method validation should include lots manufactured outside of the acceptable specification limits to demonstrate discrimination between acceptable and unacceptable lots of material. Reportable results for QC should be percent drug released. Variability should not be the primary consideration for setting specifications. A strong IVIVC with appropriate specifications will minimize the number of later studies for process changes. Currently, an official FDA or USP in vitro release method for DES products is not available. Modified compendial equipment has been deemed acceptable as part of registered specifications, with appropriate justification and validation. It is not necessary to have real-time release (due to limitations of stents, time, cost, etc.). It is necessary to ensure that in vitro test is representative. Specifications should be based on clinical, stability, and production batches and should ensure consistent performance from lot to lot.

\section{Day 2}

Speaker: Roger Williams, M.D.

\section{International Perspectives on the Drug Release of Special Dosage Forms}

Dr. Williams provided a history of the Bioequivalence panel, the interaction of the panel with agencies, and the evolution of guidances for BA and BE. The FDA guidance along with the FIP guidance use biowaivers and implementation of the BCS. He discussed the importance of comparisons and the need for calibrators to compare results. He discussed measurement units for drug release testing and the lack of an SI unit for drug release. Currently, mass versus time is the typical measurement. He reemphasized the need for USP calibrators as a publicly available reference material for comparing procedures. Next, USP chapter $<1151>$ was introduced as the general chapter to define dosage forms. This chapter is being revised, based on the Dose Form Taxonomy Stimuli article, to combine similar dosage forms, to clarify the relation of specific performance tests based on administration routes, and to append a glossary. A summary of USP general chapters for Quality and Performance for varying dosage forms was provided. The focus then shifted to performance verification testing. A summary of $<711>$ harmonization efforts was provided, and concerns about Q4B Annex 7 and the draft FDA guidance were noted. The USP does not agree with reliance on mechanical calibration alone and feels that there is a need for 
independent material to compare dissolution from different methods, instruments, and so forth. The USP has modified the nomenclature of calibrators to reference materials and looks at the materials more for proficiency testing (performance verification test) than for calibration. Historical issues with prednisone were summarized. Data from an intralaboratory variance study were presented, and these data were utilized to determine acceptance criteria for prednisone. As of December 1, 2009, salicylic acid RS tablets officially have been removed from $<711>$ as a requirement. For new lots of prednisone, geometric mean and standard deviation acceptance criteria will be used. The new performance verification recommendation has two steps that define options for the number of tablets to test. The USP believes reference material is necessary to ensure comparability across procedures.

\section{Speaker: Patrick Marroun}

In Vitro Release of Injectables - Regulatory Perspective

The focus of this presentation was modified-release injectables. All data presented as part of the presentation are available through the freedom of information act. Modified-release injectables are challenging because small levels of product are delivered over long periods of time. Current dissolution equipment is not designed for these unique methods, and modeling in vivo performance is complex. Method development is critical and is necessary to evaluate release under multiple conditions. Critical considerations include apparatus, media, physical factors, and discriminating ability. The goal for a method is to reach $80 \%$ released or a plateau and to be able to reject unacceptable lots (may require manufacture of intentionally unacceptable lots to demonstrate this). Setting specifications should be completed by considering all available data, including clinical, stability, and production batches. Specifications should be set to discriminate acceptable formulations without extreme variability. Methods and specifications should cover beginning, middle, and end. The main consideration should be release specifications, which may not be based on cumulative amounts. When determining IVIVC, there should be less than a $20 \%$ difference between $C_{\max }$ and AUC. Accelerated methods are often necessary for QC environments. The accelerated method must reflect in vivo performance with proper discriminating ability. A good method will demonstrate IVIVC by reflecting in vivo release, differentiating formulations, rejecting unacceptable lots, and ensuring appropriate product for the patient. Examples of sustained-release IVIVC were presented including Mirena, where a strong initial correlation prevented the need for clinical trials to support formulation changes. Another example presented was Nuvaring where rpm and $\mathrm{pH}$ did not impact release, providing better in vivo release predictions. The Nuvaring in vitro release data did not correlate with in vivo data, but the method was accepted by the FDA because it was capable of evaluating the process. The final example presented was Viadur, which has immediate release followed by constant release over time. For this product, the in vitro release was compared with the retained amount in vivo. It was emphasized that science and the rationale for comparison are key and that a good comparison may prevent additional clinical studies as the manufacturing process evolves. It is critical to determine important characteristics for sustained-release parenterals, to choose the appropriate conditions for in vitro release testing, to evaluate multiple formulations in vivo to ensure appropriate discriminating ability of the in vitro method, and to evaluate safety and efficacy profiles throughout comparisons. Strong method development can minimize the number of studies needed to support manufacturing or dosing changes. Strong collaboration between academia, regulatory agencies, and industry will help to standardize additional types or modification requirements for dissolution instrumentation.

Speaker:Diane Burgess, Ph.D.

\section{In Vitro Release of Injectables - Academic Perspective}

This presentation focused on in vitro release testing and particle size analysis. Before developing an in vitro release test, it is important to evaluate in vivo factors, if known. There are two types of in vivo factors: delivery system independent and delivery system dependent. In vitro release methods may need to mimic delivery system factors. The type of in vitro release method depends on the need for the method. For example, quality control methods may not be the most suitable for IVIVC, but may be capable of determining the impact of manufacturing process changes, and so forth. Other in vitro release methods may be developed to assess such things as in vivo stability and safety. While the test may not be appropriate for IVIVC, it should have biorelevance. IVIVC principles for controlled-release parenterals (liposomes or microspheres) should be developed based on in vivo relevance, and appropriate in vivo factors such as impact of tissue, available volume at the site, and motion at the site must be considered. These factors as well as end-use needs may complicate method development. There is no standard technology for in vitro release testing of controlled-release parenterals at this time. Methodologies currently employed for in vitro testing include membrane diffusion, the technique of sample and separate, in situ technique, and continuous flow technique. The difficulty of the mentioned techniques is the lack of standardization of the methodologies and thus lack of reproducibility between laboratories. Possible alternatives to the standard paddle in vitro release test include utilization of the dialysis sac versus sample and separate, employment of USP Apparatus 4 with glass beads (which help prevent agglomeration), or the addition of surfactant. While surfactant may be an option, it is not always beneficial because it may affect release rate. When Apparatus 4 is used to evaluate in vitro release from microspheres, temperature and formulation differences affect release. It is necessary to evaluate the impact of both during method development. Many times it is complicated to mimic 
complex release profiles for modified-release product through in vitro testing. One scenario is to develop a real-time test that provides IVIVC followed by an accelerated test for QC purposes. While modified in vitro release strategies may be the most beneficial, compendial apparatus should be attempted first. Some disadvantages of Apparatus 2 are microsphere aggregation, large media volumes, evaporation potential, loss of microspheres during sample manipulations, and operator variability. Fiber-optic probes may be an option to minimize sampling variability, but sticking microspheres and bubbles created from stirring are not corrected through this improvement. If a noncompendial approach proves to be the best, the method should be robust, and capabilities and advantages should be clearly described as part of registration. Apparatus 4 has been employed for multiple sustained-release parenterals that are currently marketed. Method validation should include the typical method validation parameters such as accuracy/recovery, robustness, and reproducibility (intra- and interlaboratory). Robustness is critical and should include effects of variation of cell preparation technique (glass beads, sample introduction, etc.) as well as temperature. For liposomes, the dialysis sac is the typical technique for evaluation of in vitro release. The reverse dialysis sac has also been utilized and offers advantages of continuous phase of donor chamber and larger membrane surface area. Insertion of dialysis sacs into Apparatus 4 have been evaluated to provide additional automation and efficiency. Because Apparatus 4 is compendial, there may be more opportunities for standardization across formulations including microspheres, liposomes, and suspensions.

Speaker: Mary Stickelmeyer, Ph.D.

\section{In Vitro Release of Injectables Industrial Perspective}

This presentation provided background and specific literature examples for modified-release (MR) injectables. There are multiple types of MR dosage forms marketed today. These dosage forms offer advantages of less plasma level fluctuation, fewer adverse events, improved compliance, and potentially lower doses. MR parenterals also have disadvantages that include reaction or pain at the injection site, excipient by-products, and continued drug release after the expected efficacy period. Regulatory agencies consider MR parenterals to be more complex. In vitro release tests are harder to develop and IVIVC is more complicated to establish. While regulatory guidance and external publications exist, there is not yet a standard for in vitro release testing of modified-release injectables. USP has published a stimulus article for critical quality and performance parameters for parenteral dosage forms. The article covers types of drug delivery systems, product-specific differences, and types of modified-release dosage forms. As with other presentations, the difficulty of developing an in vitro method that adequately compares in vitro to in vivo results was emphasized. It is necessary to begin method development early and to ensure the inclusion of multiple lots early in development. Early development of a robust in vitro release method may decrease the amount of work needed through formulation development. Quality by design becomes critical for in vitro release method development for modified-release dosage forms. Gathering feedback from regulatory agencies throughout development is helpful for strong submission packages. Additional attributes that must be understood for injectable dosage forms include release and administration performance. Product uses and drug delivery systems will impact development of in vitro methodology. Other tests such as particle size and distribution may be as representative of the formulation as in vitro release. Due to multiple factors, IVIVC may not be possible, and it may be feasible to use an alternate methodology to measure critical attributes of the drug product. The main key is that the manufacturing process and design space must be carefully developed and understood to develop and select the appropriate discriminating methods for process monitoring. In some cases, a real-time release test may be identified early, and an accelerated test may be developed later. Apparatus 4 offers advantages for in vitro release testing because it is a compendial method and may be more standardized for testing of MR dosage forms. Orthogonal methods may also be employed to help characterize products. Risk assessment tools should be utilized to ensure the right methods are employed to measure the correct critical process parameters.

Speaker:Thomas Redelmeier, Ph.D.

\section{Targeted Drug Delivery with Liposomes}

Liposomes provide benefits of improved solubility for sparingly soluble compounds, reduction of irritation in vivo, improved therapeutic index, reduced toxicity, targeted therapy, and intracellular delivery. Liposomes typically range from 80 to $100 \mathrm{~nm}$ and are variable in their materials depending on the requirements of the therapy. In vitro tests are developed early. Effective BE studies are more complex if the early in vitro work does not establish a thorough understanding of the mechanism for release, the importance of manufacturing process variables, pharmacodynamics, rank-order prototypes, and basic information for preclinical and clinical programs. For liposomes, additional in vitro tests may be developed to support QC, BE studies, IVIVC, and to understand degradation of lipid excipients. The mechanism of liposome drug release involves blood volume, blood serum, plasma proteins, vesicles, osmotic gradients, and cellular uptake. Release may occur as a burst or through exchange, diffusion, metabolism, or cellular uptake. There are some immediate-release liposomal products. For this type of product, understanding API behavior in vivo is critical. An in vitro release assay may not be used in a QC environment because release is independent of the material properties of liposomes. Instead, QC assays should measure precipitation of the API. Sparingly soluble 
compounds must be studied differently than immediate-release compounds. They may be evaluated with dialysis, but appropriate sink conditions and representative collisions may not be possible. For intermediate-release compounds, it may be possible to develop a real-time in vitro assay. Accelerated assays are more typical for slow-release products. Most in vitro assays monitor drug release, but it may also be necessary to rate and rank-order prototypes because not all liposomes are the same. Multiple examples describing ways to affect product release by changing liposome composition were provided. API also plays a key role in release rate. Understanding modes of delivery, impact of liposome characteristics, API properties, overall permeability, and impact of drug/lipid ratios assist in developing consistent in vitro methods. Effective quality control assays are ultimately a measure of the permeability of the microsome. The assay should provide precise, robust data over the appropriate time frame without disturbing the mechanism of release. The method should be sensitive to the initial burst and should correlate with in vivo data. It should also be appropriately discriminating. The in vitro method may be a closed system with controlled temperature, must achieve adequate sink conditions over the appropriate API concentration, and may require perturbants to induce release. Multiple separation techniques have been employed to separate the free API from the liposome and include equilibrium separation, solid phase extraction, gel filtration chromatography, and dialysis. Incubation at different temperatures has been employed to evaluate forced degradation but may not equate to in vivo release. Qualification of the in vitro release should include mass balance, precision, robustness, and specificity. As with many modified-release formulations, there is no standard test to cover all liposome formulations. Development should begin early and evolve based on clinical studies and manufacturing changes.

\section{Speaker:Horst-Dieter Friedel, Ph.D.}

\section{Conclusions and Next Steps}

This presentation summarized main points as provided by the speakers. A high-level summary of the types of modified-release dosage forms and in vitro release tests (including new and modified technologies) was provided. The path forward for the FIP dissolution group is to revise the FIP/AAPS Guidelines for Dissolution/In Vitro Release Testing of Special/Novel Dosage forms. PSWC 2010 was also introduced in this summary.

\section{Summary of Questions/Comments:}

Q: How easily can PLGA be translated to in vivo systems? For example, what is the impact of degradation if a product is injected more as a lump?

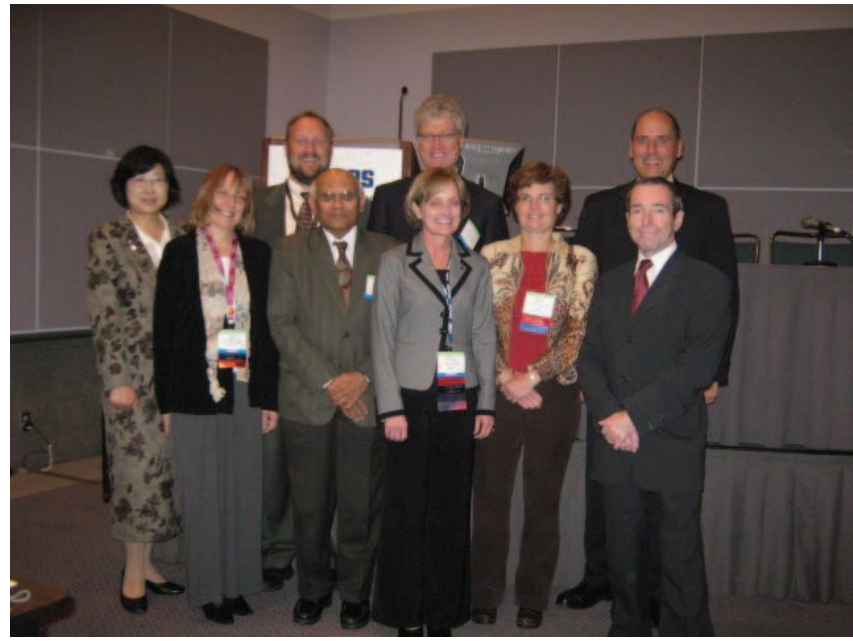

Workshop Planning Committee: From left to right-Chikako Yomota, Mary Stickelmeyer, Todd Cecil, Vinod Shah, Horst-Dieter Friedel, Cynthia Brown, Lucinda Buhse, Johannes Kraemer, Michael Morris

A: Comments were that this is important as part of development studies. It is also necessary to ensure that everything comes out of the syringe during injection.

A comment was provided that PLGA behaves like liposomes with higher molecular weights. There is an initial burst where the product releases from the surface, then a lag phase. Then there may be a secondary burst phase.

It may be possible to have mixed portions of microspheres to achieve linear release.

Q: What is the impact of glass beads in Apparatus 4? Do microspheres stick?

A: No sticking has been noted; no bead breakage has been noted. For liposomes, no Apparatus 4 experience was noted, but no adherence to glass has been observed.

Q:When are in vitro methods used for modified-release? For example, which comes first, in vitro method or in vivo study?

A: Ideally, there is an understanding of in vitro prior to in vivo tests. Then, methods should be refined when in vivo data are available.

Liposomes may require in vivo testing first because of complex interactions, differences with specific site characteristics, and so forth. Experience with in vitro methods recommends using closed vessels to minimize evaporation, determining drug stability, and determining the importance of sink conditions. Low volumes of media have been employed. Temperature is important as a critical parameter, and most methods are 4-6 h.

Q: Because of burst/lag/burst, is it okay to have two tests?

A: For IVIVC to be acceptable, it must predict the entire profile. 